\title{
A SOURCE FOR MASS-LOSS INSTABILITIES IN LBV'S - THE CASE OF P CYGNI
}

\author{
A.W.A. Pauldrach, J. Puls, R.P. Kudritzki \\ Institut f. Astronomie und Astrophysik der Universität München \\ Scheinerstr. 1, D-8000 München 80, F.R.G.
}

Since the basic mechanisms that produce photometric variations, shell ejections, and eruptions of LBV's are still unknown, it is worthwhile to investigate whether instabilities can occur when the improved self-consistent NLTE treatment of radiation-driven winds (see Pauldrach et al. 1986, Pauldrach 1987, Puls 1987, Pauldrach \& Herrero 1988) is applied to objects lying in the LBV part of the H-R diagram. The motivation is obvious: LBV's have lost considerable fractions of their initial masses and hence have $L / M$ ratios close to the Eddington limit. For such objects, radiation-driven wind theory predicts not only a strong dependence of the mass-loss rate on the self-consistently calculated parameters $\mathrm{k}, \alpha$, and $\delta$, which result from the NLTE occupation numbers of the 133 ions contributing to the line force, but also on $\Gamma\left(=\mathrm{L} / \mathrm{L}_{\mathrm{Edd}}\right): \dot{\mathrm{M}} \sim \mathrm{k}^{1 /(\alpha-\delta)}(1-\Gamma)^{(\alpha-1) /(\alpha-\delta)}$. Here we investigate the dependences of $\dot{M}$ on $M$ (through $\Gamma$ ) and on the physical environment of the atmosphere (through $\mathrm{k}, \alpha, \delta$ ) separately. The calculations are performed over a large model grid of stellar parameters for P Cyg, a typical LBV (see Fig. 1).

To study the $(1-\Gamma)$-dependence in detail, we calculated $k, \alpha, \delta$ for a low-mass model (\#5) and a high-mass model (\#1). Figure 2 shows the mass-loss rates computed using these two different sets and $\log \left(\mathrm{L} / \mathrm{L}_{\odot}\right)=5.74$ over the range of $\Gamma(\mathrm{M})$. Apart from the strong dependence of $\dot{M}$ on $(1-\Gamma)$, the most significant feature of Fig. 2 is the large gap between the curves obtained with the two model sets. It is also remarkable that the observed $\dot{M}$ for P Cyg lies between these two curves. This means that, in the parameter range applicable to $\mathrm{P}$ Cyg, a physical process that has a dramatic influence on the line force is initiated in the atmosphere. In order to investigate this behavior in more detail we have carried out fully self-consistent calculations with a constant L (see Fig. 3). Surprisingly, rather than gradual transitions from the low to the high $\dot{M}$ curve of Fig. 2, these calculations show strong discontinuities, revealing an instability mechanism for $P$ Cyg; the discontinuities occur in the observed $\dot{M}$ range and the corresponding models are close to the $\mathrm{M}-\mathrm{L}$ relation predicted by Maeder and Meynet (1987). This mechanism can be activated by small changes of either $\Delta R / R<1.5 \%$ or $\Delta L / L$ $<3 \%$. Although we do not understand how these photospheric changes occur, the

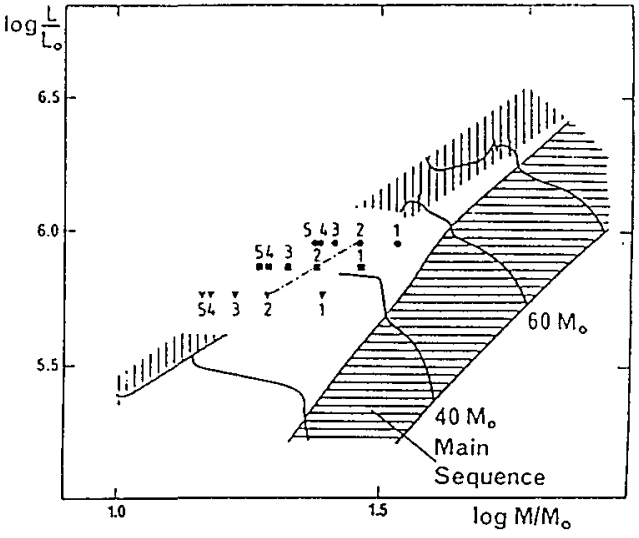

Fig. l: Mass vs. luminosity. Models numbered 2 correspond to $\log \mathrm{L} / \mathrm{L}_{\odot}=5.74,5.86,5.97$ (Lamers et al. 1985) and the masses predicted by Maeder and Meynet's (1987) tracks for $T_{\text {eff }}=19300 \mathrm{~K}$. Thus the dashed line is the $\mathrm{M}-\mathrm{L}$ relation for post-RGB objects at the temperature of P Cygni. The other models tell us the parameters if we distrust the evolutionary theory and adopt different masses at $T_{\text {eff }}=19300 \mathrm{~K}$ for constant luminosities. 

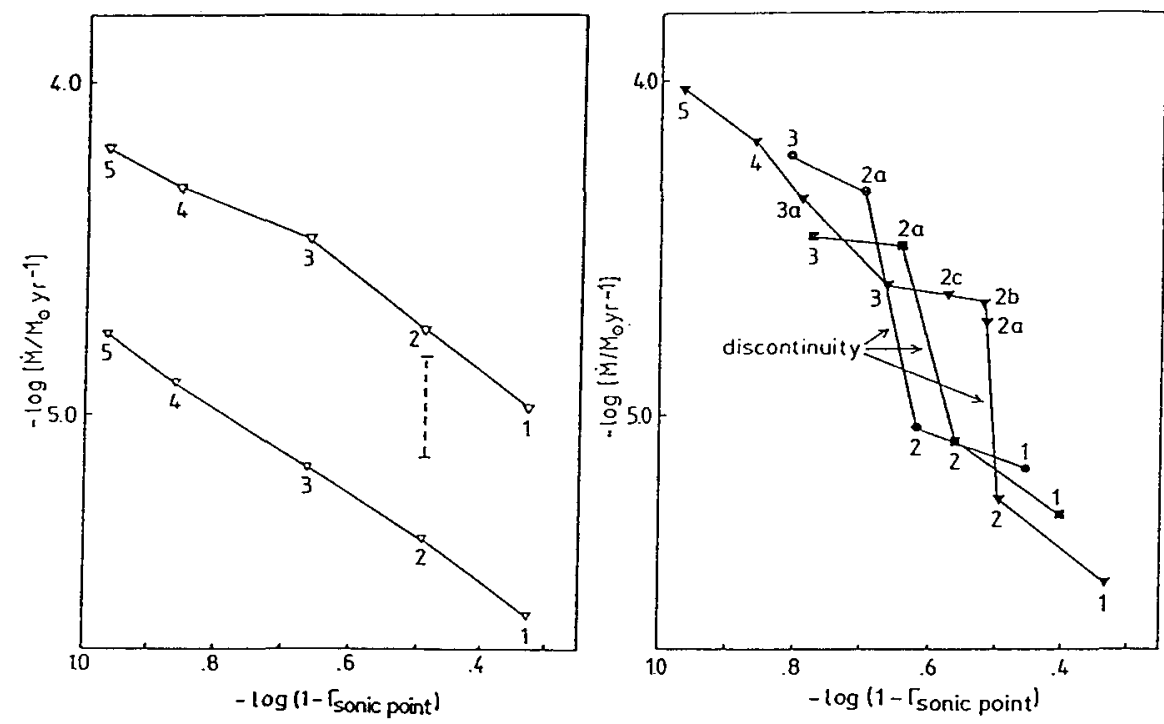

Fig. 2: Mass-loss rate vs. $(1-\Gamma)$, where the approximate sets of $\mathbf{k}, \alpha, \delta$ are kept fixed. Vertical dashed line: rate observed for P Cyg. Fig. 3: Similar plot for the tracks of constant luminosity shown in Fig. 1. Models $2 a, 2 b, 2 c$, and $3 a$ correspond to intermediate grid points. (See text.)

reason for the jump in $\dot{M}$ is clear from our calculations: the high-mass models with small $\Gamma$ exhibit moderate mass-loss rates because the Lyman continuum is optically thin and, hence, due to the strong ionizing radiation field, higher ionization stages are preferred which are less important for the line force (see Pauldrach 1987). Moving toward lower masses and higher $\Gamma, \dot{M}$ increases according to the $(1-\Gamma)$ dependence, until a critical density is reached at a certain critical $\Gamma$-value. At this critical density the Lyman continuum suddenly becomes optically thick, and consequently the reduced ionizing radiation field causes dramatic changes in the wind ionization and in the radiative line acceleration, producing the jump in mass-loss rate.

Conclusions: By means of our self-consistent NLTE calculations we have demonstrated that $P$ Cygni's wind is highly unstable with respect to small changes in photospheric radius, $\Delta R / R<1.5 \%$, or in luminosity, $\Delta L / L<3 \%$. However, the present work is only a first step. The next steps will be:

-- a study of the mass loss in various phases of P Cyg;

-- an investigation of whether this instability mechanism is a common feature of all or most of the LBV's;

-- a study of whether the instability mechanism is self-initiating in the sense that the jump in $\dot{M}$ can explain the necessary small changes of the stellar photosphere.

References: H. Lamers, M. de Groot, \& A. Cassatella 1983, Astron. Astrophys. 128, 299; A. Maeder \& G. Meynet 1987, Astron. Astrophys. 182, 243; A.W.A. Pauldrach, J. Puls, \& R.P. Kudritzki 1986, Astron. Astrophys. 164, 86; A.W.A. Pauldrach 1987, Astron. Astrophys. 183, 295; A.W.A. Pauldrach \& A. Herrero 1988, Astron. Astrophys. 199, 262; J. Puls 1987, Astron. Astrophys. 184, 227. 